\title{
Extratos de Erva-de-Santa-Maria na saúde pública: controle do vetor de arboviroses
}

\author{
Erva -de- Santa-Maria extracts in public health: vector control of arboviruses \\ Extractos de Erva-de-Santa- María en la salud pública: control del vector de arboles
}

\section{Lauriane de Assis Proença Pinto ${ }^{1 *}$, Igor Luiz Souza da $\mathrm{Cruz}^{2}$, Thiago Dutra Dias ${ }^{3}$, Ana Paula de Carvalho Faria ${ }^{4}$, Sebastião Jorge da Cunha Gonçalves ${ }^{5}$, Marise Maleck ${ }^{6}$ \\ Como citar esse artigo. Pinto, LAP; da Cruz, ILS; Dias, TD; Faria, APC; \\ Resumo} Gonçalves, SJC; Maleck, M. Extratos de Erva-de-Santa-Maria na saúde pública:controle do vetor de arboviroses. Revista Pró-UniverSUS. 2019 Jan./Jun.; 10 (1): $102-105$.

As arboviroses são uma grande preocupação na saúde pública nacional, e os programas com pouquíssima participação das comunidades, sem integração intersetorial e com pequena utilização do instrumental epidemiológico mostraram-se incapazes de conter um vetor com altíssima capacidade de adaptação ao ambiente criado pela urbanização. Há a preocupação e constantes sobre as ações desenvolvidas pelas equipes de saúde sobre as doenças causadas pelo $A$. aegypti. Este estudo buscou, através de extratos naturais de Chenopodium ambrosioides alternativas populares no controle do mosquito vetor. Nos bioensaios, foram utilizadas 20 larvas de terceiro estádio (L3), por grupo, em triplicata, totalizando 60 grupos testes e os grupos controle e testemunho. Os extratos foram aplicados na concentração de $50 \mu \mathrm{g} / \mathrm{mL}$ no meio de criação das larvas de A. aegypti. Os resultados mostraram toxicidade de $100 \%$ de larvas de terceiro estádio (L3), com o extrato hexânico obtido da extração em laboratório de química. Este dado justifica a hipótese desta planta atuar com eficácia no controle do A. aegypti e contribuir no controle das epidemias de arboviroses. Importante salientar que o enfermeiro apresenta função relevante na atenção básica no controle destas doenças. Porém, é necessário o envolvimento da população e de políticas públicas para que seja possível a real prevenção das arboviroses.

Palavras-chave:Enfermagem, Arboviroses, Saúde pública.

\begin{abstract}
Arboviruses are a major concern in national public health, and programs with very low community participation, without intersectorial integration and with little use of epidemiological instruments, have proved incapable of containing a vector with a high capacity to adapt to the environment created by urbanization. There is concern and constants about the actions developed by the health teams on diseases caused by $A$. aegypti. This study sought popular alternatives in mosquito vector control by using natural extracts of Chenopodium ambrosioides. In the bioassays, 20 larvae of third stage (L3), per group, were used in triplicate, totaling 60 test groups and the control groups. The extracts were applied at the concentration of $50 \mu \mathrm{g} / \mathrm{mL}$ in the medium of A. aegypti larvae. The results showed toxicity of $100 \%$ third instar larvae (L3), with the hexane extract obtained from the extraction in the chemistry laboratory. These data justify the hypothesis of this plant to act effectively in the control of A. aegypti and to contribute to the arbovirus epidemics control. It is important to emphasize that the nurse has a relevant role in primary care in the control of these diseases. However, the involvement of the population and of public policies is necessary so that the real prevention of arboviruses be possible.
\end{abstract}

Keywords: Nursing, Arboviruses, Public Health.

\section{Resumen}

Los arbovirus son una preocupación importante enlasalud pública nacional, y los programas com muy poca participación comunitaria, sin integración intersectorial y conpoco uso de instrumentos epidemiológicos, han demostrado ser incapaces de contener un vector con una alta capacidad de adaptación al entorno creado por la urbanización. Existe preocupación y constantes sobre lãs acciones desarrolladas por los equipos de salud sobre lãs enfermedades causadas por $A$. aegypti. Este estúdio buscó alternativas populares em El control de mosquitos vectores mediante el uso de extractos naturales de Chenopodium ambrosioides. Em los bioensayos, se utilizaron 20 larvas de latercera etapa (L3), por grupo, por triplicado, conun total de 60 grupos de prueba y los grupos de control. Los extractos se aplicaron a la concentración de $50 \mu \mathrm{g} / \mathrm{ml}$ enelmedio de larvas de $A$. aegypti. Los resultados mostraron una toxicidaddel 100\% de larvas Del tercer estadio (L3), conelextracto de hexano obtenido de la extracción em el laboratorio de química. Estos datos justifican la hipótesis de esta planta para actuar eficazmente en el control de A. aegypti y contribuir al control de las epidemias de arbovirus. Es importante enfatizar que la enfermera tiene un papel relevante em la atención primaria em el control de estas enfermedades. Sin embargo, la participación de la población y de las políticas públicas es necesaria para que la prevención real de los arbovirus se a posible.

Palabras clave: Enfermería; Arbovirus; Salud pública.

Afiliação dos autores: . 1Acadêmica do Curso de Enfermagem, Universidade de Vassouras. RJ, Brasil. ORCID: https://orcid.org/0000-0002-5993-6298

2. Mestre. Laboratório de Insetos Vetores e Mestrado Profissional em Ciências Ambientais da Universidade de Vassouras. RJ, Brasil. ORCID: https://orcid.org/0000-0002-7376-6184

3. Especialista. Laboratório de Insetos Vetores, Universidade de Vassouras. RJ, Brasil. ORCID: https://orcid.org/0000-0002-8998-3175

4. Acadêmica do Curso de Engenharia Química, Laboratório de Química, Universidade de Vassouras. RJ, Brasil. E-mail: anapaula.carvalhofaria@gmail.com; ORCID: https://orcid. org/0000-0001-6231-9250.

5. Mestre. Professor do Curso de Enfermagem, Pró-Reitoria de Ciências da Saúde; Curso de Medicina, Pró-Reitoria de Ciências Médicas, Universidade de Vassouras. RJ, Brasil. ORCID: https://orcid.org/0000-0002-4228-4641

6. Doutora. Professora do Curso de Medicina, Pró-Reitoria de Ciências Médicas; Laboratório de Insetos Vetores e Mestrado Profissional em Ciências Ambientais da Universidade de Vassouras. RJ, Brasil; Pesquisadora do Laboratório de Entomologia Médica e Forense, IOC, FIOCRUZ, Rio de Janeiro, RJ, Brasil. ORCID: https://orcid.org/0000-0001-7699-7896

* Email de correspondencia: laurianeproenca@gmail.com

Recebido em: 01/12/18. Aceito em: 25/04/19. 


\section{Introdução}

Diante dos desafios em relação às arboviroses delineado pela expansão destes vírus em todo $\mathrm{o}$ mundo, torna-se imprescindível a adoção de estratégias específicas, com maiores investimentos em métodos adequados, que forneçam sustentabilidade às ações estabelecidas pelas redes de vigilância, além de ensejarem a análise de sua efetividade. Assim, em face do atual cenário de surtos e epidemias de zika, chikungunya e dengue, conhecer o efeito larvicida de produtos naturais pode ser uma alternativa viável no controle do transmissor destas doenças.

O mosquito Aedes aegypti é uma das 500 espécies do gênero Aedes (Diptera: Culicidae). É um mosquito encontrado em áreas urbanas, vetor de doenças como dengue, zika, chinkungunya e febre amarela urbana. A sua grande capacidade de adaptação a condições adversas, tais como períodos de quiescência de ovos em ambientes inóspitos, e o crescimento normal em águas poluídas, faz com que o controle deste vetor seja muito difícil. Quando os ovos entram em contato com a água, após um ciclo de dois a quatro dias, originamse as larvas que possuem quatro estádios: L1, L2, L3 e L4. Posteriormente originam-se as pupas, e após os adultos. ${ }^{1} \mathrm{O}$ controle populacional dos mosquitos tem se concentrado nas fases larvais, em que o processo é mais eficiente, mais seletivo, e menos oneroso aos programas de prevenção à dengue. ${ }^{2}$

Dentre as estratégias de controle deste vetor, ainda é muito utilizado em grande escala, inseticidas mais agressivos, como por exemplo, os organofosforados. A utilização deste inseticida pode causar vários danos ao meio ambiente, como intoxicação e contaminação ambiental, o que ocasiona riscos à biodiversidade local e também à saúde humana ${ }^{3}$. O seu uso contínuo e indiscriminado pode ocasionar a seleção de população de vetores resistentes, contribuindo para o surgimento de novas pragas. ${ }^{1}$

Vários estudos têm chamado a atenção para os produtos naturais com atividade larvicida que poderiam ser úteis no controle de diversos vetores ${ }^{4,5,6,7}$ incluindo o A. aegypti ${ }^{8,9}$. As plantas são fontes riquíssimas de substâncias farmacologicamente ativas, e algumas delas podem apresentar efeitos inseticidas e larvicida para o controle de insetos vetores para a saúde pública e para culturas agrícolas. O controle de vetores com o uso de produtos naturais é menos impactante em relação aos inseticidas convencionais, pois esses produtos apresentam menos impacto do meio ambiente e menor toxicidade sobre as espécies não-alvo ${ }^{2}$. Vários pesquisadores têm trabalhado com produtos naturais, visando obter extratos de plantas, com atuação no desenvolvimento embrionário, na fase larval, fase de pupa e na emergência dos adultos de insetos, inclusive do $A$. aegypti ${ }^{8,9}$.

A Chenopodium ambrosioides L. da família Amaranthaceae, também conhecida como erva-desanta-maria, ou mastruz, é encontrada em todas as regiões do Brasil, sendo originária do México. Seus extratos ou óleo essencial apresentaram propriedades acaricida e inseticida tendo a maior concentração isoladamente presente em suas folhas ${ }^{10}$. Na medicina popular é utilizada como estomáquica, antiespasmódica, emenagoga e vermífuga. Sua maior utilização, no saber popular, é como vermífuga, e de acordo com a com a Organização Mundial de Saúde (OMS) a erva de Santa Maria é um dos fitoterápicos mais utilizados no mundo inteiro, com essa finalidade ${ }^{11}$.

Estudos com extratos e óleos essenciais de Ervade-santa-maria, vêm sendo realizados também no setor agropecuário, e se mostra, por exemplo, como acaricida no controle de teleóginas de carrapatos bovinos ${ }^{12}$.

Diante das inúmeras propriedades desta planta, este estudo buscou avaliar a atividade larvicida dos extratos de $C$. ambrosioides L. no controle de $A$. aegypti, vetor de arboviroses.

\section{Metodologia}

Estudo explicativo. Como procedimento utilizou-se a experimentação científica, e o método quantitativo.

A extração do material vegetal foi realizada nos Laboratório de Química e no Laboratório de Insetos Vetores, ambos da Universidade de Vassouras.

\section{$1^{\mathrm{a}}$ etapa}

Para obtenção dos extratos alcoólicos de $C$. ambrosioides foi feita a trituração e maceração de $5 \mathrm{~g}$ da planta (caule, sementes e folhas) com $100 \mathrm{~mL}$ de cada solvente orgânico: metanol, etanol, acetato de etila e hexano. A extração foi realizada em temperatura ambiente. Após este processo, os extratos foram armazenados em vidro âmbar e colocados para descansar por oito dias.

\section{$2^{\text {a }}$ Etapa}

Os extratos da Erva-de-santa-maria foram realizados em um aparato Soxhlet, e a planta foi envolvida em papel filtro. A extração foi realizada utilizando $15 \mathrm{~g}$ da planta (caule, folha e sementes) e $700 \mathrm{~mL}$ de cada solvente. Fixou-se um tempo de $1 \mathrm{~h}$ para obtenção dos extratos para cada solvente. O extrato de acetato de etila apresentou $1,357 \mathrm{~g}$ com rendimento de 9 , $05 \%$; o extrato metanólico $1,179 \mathrm{~g}$ com rendimento de 7 , $86 \%$; o extrato hexânico com $0,3088 \mathrm{~g}$ e rendimento de $2,058 \%$; e o extrato etanólico com $1,514 \mathrm{~g}$ e rendimento 
de $10,09 \%$.

\section{Bioensaios}

Os bioensaios foram realizados no Laboratório de Insetos Vetores da Universidade de Vassouras.

Os ovos foram obtidos do Laboratório de Transmissores de Hematozoários e Núcleo Operacional Sentinela de Mosquitos Vetores/IOC/FIOCRUZ, Rio de Janeiro.

Os bioensaios foram realizados em meio de criação das larvas contendo água mineral $(20 \mathrm{~mL})$ contendo 20 larvas em terceiro estádio (L3) em triplicatas (R1, R2, R3) totalizando 60 larvas por grupo, e com três repetições. Os extratos foram aplicados no meio de criação das larvas no volume de $20 \mu \mathrm{L}$ dos extratos para $20 \mathrm{~mL}$ de água, em uma proporção de 1:15,9,13,14 adaptado de acordo com a World Health Organization ${ }^{15}$ para os grupos testes; o grupo testemunho foi colocado apenas o solvente de diluição e o grupo controle não foi aplicado nenhum solvente ou extrato.

Após $1 \mathrm{~h}$ de tratamento, as larvas foram alimentadas com dieta normal $(0,3 \mathrm{mg} /$ larva $)$ de ração de peixe (Alcon Basic ${ }^{\circledR}$ ) e mantidas em câmara climatizada BOD a $27^{\circ} \mathrm{C} \pm 1$ e UR $70 \% \pm 10$. O bioensaio foi observado até a total mortalidade.
Os dados foram submetidos ao teste de Tukey (significância de 5\%) pelo software Graphpad-Prism ${ }^{16}$; a análise de variância (ANOVA) ${ }^{17}$ e desvio padrão calculado de acordo com as médias.

\section{Resultados}

O tratamento das larvas de $A$. aegypti com os extratos em estudo mostrou mortalidade de $100 \%(2,0$ $\pm 0,2^{* * *}$ ) das larvas de terceiro estádio (L3) com o extrato hexânico. Este extrato foi obtido por processo de extração fitoquímica. O mesmo extrato realizado manualmente apresentou apenas 5\% de mortalidade de larvas de terceiro estádio (L3) (11 \pm 0$)$ e $45 \%$ de mortalidade $\left(20,6 \pm 3,7^{* *}\right)$ das larvas de quarto estádio (L4) (Tabela 1).

Os demais extratos de C. ambrosioides apresentaram mortalidade abaixo de $70 \%$ para larvas L3 (Tabela 1).

\section{Discussão}

Estudos demonstraram a atividade larvicida de óleo essencial de C. ambrosioides. ${ }^{18}$ Esta espécie atuou como um potente inseticida em larvas de A. aegypti

Tabela 1. Mortalidade de larvas de Aedes aegypti (L3) tratadas com extratos de Chenopodium ambrosioides na concentração de $50 \mu \mathrm{g} / \mu \mathrm{L}$.

\begin{tabular}{|c|c|c|c|c|}
\hline \multirow{2}{*}{$\begin{array}{c}\text { Tratamento } \\
\text { Etanol }\end{array}$} & \multirow{2}{*}{$\begin{array}{l}\text { Larvas L3 } \\
\mathrm{X} \pm \mathrm{SD}\end{array}$} & \multicolumn{3}{|c|}{ Larvas L4 } \\
\hline & & $\%$ & $\mathrm{X} \pm \mathrm{SD}$ & $\%$ \\
\hline Controle & $13,2 \pm 3,3$ & 18,3 & $15 \pm 3,5$ & 33,3 \\
\hline Testetanol & $3,5 \pm 1,1$ & 20 & $19 \pm 10,2$ & 52 \\
\hline Extracão Lab & $8 \pm 0$ & 11,7 & $19 \pm 6,4$ & 39,6 \\
\hline Extracão simples & $5,6 \pm 5,2$ & 36,6 & $19,3 \pm 3,8$ & 50 \\
\hline Tratamento & Larvas L3 & & Larvas L4 & \\
\hline Metanol & $\mathrm{X} \pm \mathrm{SD}$ & $\%$ & $\mathrm{X} \pm \mathrm{SD}$ & $\%$ \\
\hline Controle & $13,2 \pm 3,3$ & 18,3 & $15 \pm 5,6$ & 33,3 \\
\hline Testmetanol & $7,2 \pm 3,8$ & 8,3 & $20 \pm 5,6$ & 58 \\
\hline Extracãa Lab & $8,4 \pm 2,5$ & 35 & $13,4 \pm 3,6 * *$ & 48,7 \\
\hline Extracão simples & $10,9 \pm 3,1$ & 25 & $16,6 \pm 3,7$ & 29 \\
\hline Tratamento & Larvas L3 & & Larvas L4 & \\
\hline Hexano & $\mathrm{X} \pm \mathrm{SD}$ & $\%$ & $\mathrm{X} \pm \mathrm{SD}$ & $\%$ \\
\hline Controle & $13,2 \pm 3,3$ & 18,3 & $15 \pm 5,6$ & 33,3 \\
\hline Test. hexane & $10,5 \pm 3,3$ & 28,3 & $13,3 \pm 5,1$ & 39,5 \\
\hline Extracão Lab & $2,0 \pm 0,2 * * *$ & 100 & $0 * * *$ & - \\
\hline Extracão simples & $11 \pm 0$ & 5 & $20,6 \pm 3,7^{* *}$ & 45,6 \\
\hline Tratamento & Larvas L3 & & Larvas L4 & \\
\hline Acetato de etila & $\mathrm{X} \pm \mathrm{SD}$ & $\%$ & $\mathrm{X} \pm \mathrm{SD}$ & $\%$ \\
\hline Controle & $13,2 \pm 3,3$ & 18,3 & $15 \pm 5,6$ & 33,3 \\
\hline Testacetato & $6,9 \pm 3,9$ & 35 & $22,6 \pm 3,0$ & 67 \\
\hline Extração Lab & $6,5 \pm 2,5$ & 62 & $12,2 \pm 0,4 * *$ & 22 \\
\hline Extração simples & $7,3 \pm 1,6$ & 10 & $19,1 \pm 4$ & 68,5 \\
\hline
\end{tabular}

Experimentos com 20 larvas (L3) de A. aegypti para cada grupo teste, controle e testemunho em triplicata $(n=60)$. Média e desvio padrão $(X \pm S D)$. Significância de acordo com o teste de Tukey representados $* * * \mathrm{p} \leq 0.001 ; * * \mathrm{p} \leq 0.01 \%$ de mortalidade. 
e Anopheles arabiensis (importante vetor da malária na África Subsaariana), apresentando bons índices de mortalidade em baixas concentrações.

Testes utilizando o extrato etanólico de $C$. ambrosioides em fases imaturas de Aedes albopictus apresentaram uma $\mathrm{LC}_{50}$ entre $124.55 \mathrm{ppm}$ (larvas de primeiro instar) até $237,06 \mathrm{ppm}$ (pupas), com uma metodologia para obtenção dos extratos semelhante à deste trabalho. ${ }^{19}$

Extrato de folhas desta espécie se mostrou eficaz no controle de larvas do vetor Culex quinquefasciatus, responsável pela transmissão de filariose no nordeste do Brasil. O extrato metanólico apresentou uma mortalidade de $100 \%$ na concentração de $25 \mathrm{mg} / \mathrm{l}$ e de aproximadamente $42 \%$ na concentração de $5 \mathrm{mg} / \mathrm{l}^{20}$

Outra aplicação importante da Erva-de-santamaria é como repelente para picadas de mosquitos. A literatura aponta o impacto do óleo essencial desta planta (na concentração de 1\%) como protetor contra fêmeas de $A$. albopictus, com um índice de proteção de $53,5 \%$, permitindo repelência intermediária. ${ }^{21}$

Dentro deste contexto, é possível observar que esta planta apresenta diversas vertentes de combate contra os vetores causadores de patologias nocivas para os seres humanos. Estudos com o óleo essencial das folhas desta espécie foi diluído em uma solução aquosa de DMSO $0,1 \%$ para obtenção de diversas concentrações $(10,20$, 40,80 e $100 \mathrm{ppm}$ ) nos intervalos de 2, 4, 6, 12 e 24 horas a fim de avaliar a atividade moluscicida do óleo. A dose letal foi observada em $6 \mathrm{~h}$ nas concentrações de 80 e 100 ppm. ${ }^{22}$

Neste estudo observou-se que os extratos de C. ambrosioides diferiram quanto o percentual de mortalidade das larvas de A. aegypti. Os extratos metanólico, hexânico e de acetato de etila foram mais eficazes quanto ao efeito larvicida quando extraídos pelo processo fitoquímico. Enquanto, que o extrato etanólico obtido por extração manual mostrou-se mais eficaz em comparação ao obtido por processo fitoquímico, sobre as larvas L3 e L4 do mosquito, o que sugere a possibilidade das plantas como sendo indispensáveis ao uso popular.

\section{Conclusão}

O extrato hexânico apresentou maior significância na mortalidade das larvas de terceiro estádio com o percentual máximo de $100 \%$ de mortalidade em $24 \mathrm{~h}$, mostrando o seu potencial larvicida. Este dado propõe C. ambrosioides uma ótima opção sob o ponto de vista ecológico, como grande promissora no controle de insetos transmissores das arboviroses. Neste estudo observou-se a possibilidade do extrato etanólico como uso popular sobre as larvas desses culicídeos, representando uma alternativa acessível à comunidade.
Contudo, as arboviroses são um crescente problema na saúde pública consolidado pelo controle ineficaz do mosquito vetor transmissor dessas doenças. Cabe aos enfermeiros promotores de saúde, traçar estratégias no controle deste vetor com a finalidade de reduzir os números de agravos por arboviroses sendo um importante aliado da população no combate a essas doenças.

\section{Agradecimentos}

Os autores agradecem a Fundação de Amparo a Pesquisa do Estado do Rio de Janeiro (FAPERJ) pelo financiamento e suporte ao projeto de pesquisa "Novas estratégias para o controle do mosquito Aedes aegypti, vetor da Dengue, Chikungunya e do vírus Zika: uma abordagem integrada/RedeZIKA\#I) e ao Núcleo Operacional Sentinela de Mosquitos Vetores/IOC/ FIOCRUZ pelos ovos de A. aegypti.

\section{Referências}

1. Consoli RAGB, Oliveira RL. Principais mosquitos de importância sanitária no Brasil. Rio de Janeiro: FIOCRUZ, 1994, p. 228. Disponível em: http:// static.scielo.org/scielobooks/th/ pdf/consoli-9788575412909.pdf 10

2. Barreto CF. Aedes aegypti - Resistência aos inseticidas químicos e as novas alternativas de controle. Rev Eletrônica Faculdade Montes Belos, Goiás, 2005;1(2):62-73.

3. Almança CCJ. Extrato hidroetanólico de erva-de-santa-maria (Chenopodium ambrosoides L.) no controle do carrapato de bovinos [Rhipicephalus (Boophilus) microplus Canestrini, 1888 (Acari: Ixodidae)]. Dissertação (Mestrado em Ciências Veterinárias) - Centro de Ciências Agrárias da Universidade Federal do Espírito Santo, 2011.

4. Park IK, Schin SC, Park JD, Ahn YJ. Larvicidal activity of isobutylamides identified in Piper nigrum fruits against three mosquito species. J Agric Food Chem, 2002, .50:1866, 1870.

5. Maleck M, Santos FCC, Serdeiro MT, Guimarães AE, Ferreira B, Gunaydin K, Almeida AP. Kellin: a furanochromone with toxicity against Oncopeltus fasciatus (Hemiptera) and Aedes aegypti (Diptera). J Nat Pharma, 2013;4(1):32-6.

6. Brunherotto R, Vendramim JD. Bioatividade de Extratos Aquosos de Melia azedarach L. Sobre o Desenvolvimento de Tuta absoluta (Meyrick) (Lepidoptera: Gelechiidae) em Tomateiro. Neotrop Entomol, 2001; 30(3):455-9.

7. Valladares G, Defago MT. Palacios, S. Laboratory evaluation of Melia azedarach (Meliaceae) extracts against the Elm Leaf Beetle (Coleoptera: Chrysomelidae). J Econ Entomol, 1997; 90(3):747-50.

8. Coria C, Almiron W, Valladares L, Carpinella C, Ludueña F, Defago H et al. Larvicide and oviposition deterrent effects of fruit and leaf extracts from Melia azedarach L. on Aedes aegypti (L.) (Diptera: Culicidae). Bioresour Technol, 2008; 99: 3066-70.

9. Cabral MMO, Alencar JÁ, Guimarães AE, Kato MJ. Larvicidal activity of grandisin against Aedes aegypti. J Am Mosq Control Assoc, 2009;25(1):103-5.

10. Chiasson H, Vincent C, Bostanian NJ. Insecticidal Properties of a Chenopodium - Based Botanical. J Econ Entomol, 2004; 97(4):1378-83.

11. NVISA. Agência Nacional de Vigilância Sanitária (2011). Formulário de fitoterápicos. Farmacopéia Brasileira. Disponível em: http://www.anvisa. gov.br. Acesso em: 16. Set. 2018 
12. Borges LMF, Sousa LAD, Barbosa CS. Perspectives for the use of plant extracts to control the cattle tick Rhipicephalus (Boophilus) microplus. Rev Bras Parasit Vet, 2011; 20 (2): 89-96.

13. Narciso JOA, Soares ROA, Mallet JRS, Guimarães AE, Chaves MCO, Barbosa-Filho JM et al. 2014. Burchellin: study of bioactivity against Aedes aegypti. Parasit Vectors. 7: 172.

14. Leite ACCF, Kato MJ, Soares ROA, Guimarães AE, Santos-Mallet JR, Cabral MMO. Grandisin caused morphological changes larval and toxicity on Aedes aegypti. Rev Bras Farmacog 2012; 22 (3): 517-521.

15. [WHO] World Health Organization. Guidelines for laboratory and fieldtesting of mosquito larvicides. 2005. Disponível em :http://apps.who.int/iris/ bitstream/10665/69101/1/WHO_CDS_WHOPES_GCDPP_2005.13.pdf.

16. Motulsky H. Analyzing data with GraphPad prism. GraphPad Software Incorporated, 1999.

17. Sokal RR, Rohlf FJ. Biometry. 3rd ed., W.H. Freeman, New York. 1995. 18. Massebo F, Tadesse M, Bekele T, Balkew M, Gebre-Michael T. Evaluation on larvicidal effects of essential oils of some local plants against Anopheles arabiensis Patton and Aedes aegypti Linnaeus (Diptera, Culicidae) in Ethiopia. Afr J Biotechnol, 2009;8(17): 4183-88.

19 Subramaniam J, Murugan K, Jebanesan A, Pontheckan P, Dinesh D, Nicoletti M, et al. Do Chenopodium ambrosioides-Synthesized Silver Nanoparticles Impact Oryzias melastigma Predation against Aedes albopictus Larvae? J Clust Sci, 2017;28(1):413-36.

20. Rajkumar S, Jebanesan A. Bioactivity of Chenopodium ambrosioides L. (Family: Chenopodiaceae) against the filariasis vector Culex quinquefasciatus Say (Diptera: Culicidae). Can J Pure Appl Sci, 2008;2(1): 129-32.

21. Bueno VS, Andrade CFS. Avaliação preliminar de óleos essenciais de plantas como repelentes para Aedes albopictus (Skuse, 1894) (Diptera: Culicidae). Rev Bras Plantas Med, Botucatu, 2010; 12(2):215-19.

22. Frank HO, Vieira DF, Quadros IPS, Pinheiro PF, Queiroz VT, Junior OSP et al. Atividade moluscicida do óleo da erva-de-santa-maria (Chenopodium ambrosioides L.) sobre o molusco do gênero Biomphalaria. In: XV Encontro Latino Americano de Iniciação Científica e XI Encontro Latino Americano de Pós-Graduação e V Encontro Latino Americano de Iniciação Científica Júnior, 2011, Espírito Santo, Anais Universidade do Vale do Paraíba, 2011, p.1-4. 